\title{
Lipid-lowering drug use in Italian primary care: effects of reimbursement criteria revision
}

\author{
G. Trifirò • M. Alacqua $\cdot$ S. Corrao $\cdot$ S. Moretti $\cdot$ \\ D. U. Tari • M. Galdo • UVEC Group •A. P. Caputi • \\ V. Arcoraci
}

Received: 27 June 2007 / Accepted: 27 December 2007 / Published online: 22 January 2008

(C) Springer-Verlag 2008

\begin{abstract}
Objective To assess whether the prescribing pattern of lipid-lowering drugs (LLD) changed after reimbursement criteria revision in a general practice in southern Italy.

Methods From the Caserta-1 Local Health Service database, 93 general practitioners (GPs) who had consistently sent data about their patients during the years 2003-2005 were recruited. Prevalence of use and incidence of new treatments were calculated for each year, stratified by three drug cohorts: statins, omega-3 fatty acids, and fibrates. Subanalyses by gender, age, and indication of use were performed.

Results Overall, 1-year prevalence of LLD use increased from 2003 to 2004. After reimbursement criteria revision (November 2004), a slight decrease was observed for statins, from 41.1 (95\% CI: 39.9-42.2) per 1,000 inhabitants in 2004 to 40.3 (39.2-41.5) in 2005, while omega-3 utilization fell markedly: 14.6 (13.9-15.3) vs. $5.4(5.0-5.8)$.
\end{abstract}

G. Trifirò $(\bowtie) \cdot$ M. Alacqua $\cdot$ A. P. Caputi $\cdot$ V. Arcoraci Department of Clinical and Experimental Medicine and Pharmacology, Pharmacology Unit, University of Messina, Via Consolare Valeria-Gazzi, 98125 Messina, Italy

e-mail: trifirog@unime.it

\section{S. Corrao}

Biomedical Department of Internal Medicine \& Subspecialties, University of Palermo,

Palermo, Italy

S. Moretti · D. U. Tari • M. Galdo · UVEC Group

Caserta 1 Local Health Service,

Caserta, Italy

G. Trifirò · A. P. Caputi

IRCCS Centro Neurolesi "Bonino-Pulejo",

Messina, Italy
The use of both statins and omega-3 fatty acids was reduced particularly for primary prevention. On the other hand, utilization of statins increased in diabetic patients and as secondary prevention from 2004 to 2005. Concerning individual molecules, 1-year prevalence of use of any statin declined from 2004 to 2005, except for rosuvastatin.

Conclusions Revision of reimbursement criteria led to significant changes in the trend in LLD use in general practice in southern Italy: (1) statin utilization was slightly reduced in 2005, although it increased in certain categories, such as diabetic patients, and (2) omega-3 fatty acid use was strongly reduced even though a higher use in postinfarction cases was reported.

Keywords Lipid-lowering drug · Statins .

Omega-3 fatty acids $\cdot$ Prevalence of use $\cdot$ General practice

\section{Introduction}

Hypercholesterolemia is a well-known risk factor of cardiovascular (CV) diseases and its successful treatment with lipid-lowering drugs (LLDs) is one of the most important goals in CV disease prevention [1].

Utilization of statins has been progressively increasing worldwide since their marketing in the 1980s, supported by scientific evidence showing both their effectiveness [2,3] and good safety profile [4] in CV disease prevention.

In addition to statins, fibrates and omega- 3 polyunsaturated fatty acids are the main LLDs currently marketed. Omega-3 fatty acids, naturally available in fish, shellfish, soya oil, leafy vegetables, walnuts, and others, have been claimed to have a protective role against coronary ischemic diseases due to lowering of serum triglycerides, platelet aggregation inhibition, endothelial function modulation, 
blood pressure regulation, and anti-inflammatory and antiarrhythmic effects [5].

In Italy omega-3 fatty acids have been marketed since 2001 as prescription drugs totally reimbursed by the National Health System (NHS), while they are considered dietetic integrators in other Western countries. As a result, the consumption of omega-3 fatty acids strongly increased in Italy, moving from 722nd place in terms of national drug expenditure in 2001 to 8th in 2004 [6].

In November 2004, the Italian Drug Agency revised reimbursement criteria of LLDs, as reported in Nota 13 [7], according to updated scientific evidence.

The aim of this study was to assess whether the trend of lipid-lowering drug use changed after reimbursement criteria revision in a general practice in southern Italy. For this reason, this drug utilization study explored the years 2003-2005.

\section{Methods}

\section{Setting}

The investigation was carried out using data that were extracted from the Arianna database in the years 20032005. The database was set up by the Local Health Service of the city of Caserta in the year 2000. It currently contains information on almost 300,000 individuals who are living in the catchment area of Caserta and registered on the lists of 225 general practitioners (GPs). This sample of physicians accounts for $74 \%(225 / 305)$ of all the GPs practicing in the same area. In general, participating GPs record data during their daily clinical practice through dedicated software and, monthly, they send complete and anonymous clinical data about their patients to the Arianna database. The information collected includes patient demographics, drug prescriptions (reimbursed by NHS) coded according to the Anatomical Therapeutic Chemical (ATC) classification system, and medical diagnoses coded by the International Classification of Diseases, 9th revision, Clinical Modification (ICD-9 CM) that are linked to any drug prescription.

All the GPs enrolled in the Arianna database had previously received extensive training in data collection techniques. A number of data quality checks are routinely performed, including analysis of several variables such as monthly continuity of data submission, missing patient codes, and number of prescriptions filled daily. Any variation beyond the defined range is investigated and the data are returned to the participating GP in order to receive immediate feedback about data quality and completeness. GPs failing to meet these standard quality criteria are not retained within the analyses, according to basic standards for the conduct of pharmacoepidemiological studies [8]. So far, the Arianna database has been shown to provide accurate and reliable information on drug utilization [9-11].

Study population

Overall, 93 GPs that consistently sent reliable data to the Arianna database during the years 2003-2005 were selected for this investigation. Out of the approximately 120,000 individuals registered in their lists, users of LLDs were identified. LLD users were defined as individuals who received at least one lipid-modifying agent (ATC: C10) prescription during the observation years. Patients aged 18 years and over were included in the study, irrespective of whether LLD treatment was initiated by GPs or by specialists. Indeed, in Italy outpatients who receive prescriptions from specialists working in the public or private health-care services get the medicines free of charge only through GP prescriptions. After identification of LLD users, the following information was retrieved using the Arianna database: patient demographics, LLD-related data, including product name, dispensed quantity, indication of use, and concurrent diseases being pharmacologically treated during the years 2003-2005.

Annual utilization of different LLD types was stratified into primary or secondary prevention. A treatment was defined as secondary prevention if the LLD user was affected by coronary heart disease, cerebrovascular disease, peripheral arterial disease, $\mathrm{CV}$ procedures, or diabetes prior to the first LLD prescription date.

\section{Health policy intervention}

LLD reimbursement criteria (Nota 13) were revised in November 2004. Briefly, the main changes made by this health-policy intervention are reported below:

1. Omega-3 fatty acids are reimbursed only for treatment of hyperlipidemia in patients with prior myocardial infarction (MI), while they had been totally reimbursed before the revision.

2. Statins are reimbursed in case of hypercholesterolemia that cannot be corrected through diet only, for both primary and secondary $\mathrm{CV}$ disease prevention.

3. For primary prevention, statin use is now restricted to high-risk users, based on a national risk profile $[12,13]$. For secondary prevention, statin use is reimbursed for patients affected by coronary heart disease, cerebrovascular disease, peripheral arterial disease, $\mathrm{CV}$ procedures and, after this health policy intervention, the use of statins was also extended to patients affected by diabetes.

Fibrates, statins, and omega-3 fatty acids are all reimbursable in case of familial hyperlipidemia $(\mathrm{FH})$, as indicated also in the previous reimbursement criteria. 
Study drugs

The following drug cohorts were identified:

1. Statins: simvastatin (C10AA01), pravastatin (C10AA03), fluvastatin (C10AA04), atorvastatin (C10AA05), and rosuvastatin (C10AA07)

2. Omega-3 fatty acids (C10AX06)

3. Fibrates: clofibrate (C10AB01), bezafibrate (C10AB02), gemfibrozil (C10AB04), and fenofibrate (C10AB05)

Patients that were treated only with bile acid sequestrants, nicotinic acid and derivatives, or other lipidmodifying agents were not taken into account in this analysis since these drugs are not reimbursed according to the new health policy intervention.

In addition, lovastatin (C10AA02) and simvastatinezetimibe combination (C10BA02) were not considered because they were introduced onto the market at the end of 2005 and no prescriptions were filled by physicians within the observation period.

One-year prevalence and incidence of use

Annual prevalence of LLD treatment was calculated as the number of LLD users divided by the number of subjects alive and registered in the GPs' lists in any study year. We defined a "new user" as a patient receiving a first LLD prescription within the observation years without any recorded LLD prescription in the previous year. The 1-year cumulative incidence was calculated as the number of new users divided by the number of subjects who did not take lipid-lowering drugs in the previous year.

Both prevalence and incidence per year were expressed as rates per 1,000 inhabitants, together with $95 \%$ confidence interval (CI).

\section{Statistical analysis}

Two-tailed chi-squared test for proportions and Student's $t$ test for continuous variables, with a significance level of $P<$ 0.05 , were used for assessing the differences among users of various LLD types. Chi-square for trend was used to evaluate any significant $(P<0.05)$ variation in 1 -year prevalence of LLD use during the study years. Statistical analyses were performed using STATA 6.0 (STATA, TX).

\section{Results}

Prevalence of LLD use

Prevalence of LLD use per 1,000 inhabitants, stratified by drug types and calendar years, is shown in Fig. 1. The most commonly used LLDs are statins, which account for more than $60 \%$ of LLD use. Rate of statin users significantly ( $P<$ 0.05 ) increased from 35.3 (34.3-36.4) per 1,000 in 2003 to $41.1(39.9-41.4)$ in 2004, while it slightly decreased in the following year (40.3, 39.2-41.4).

Concerning omega-3 fatty acid use, a significantly decreased use was reported after reimbursement criteria revision: from $14.6(13.9-15.2)$ per 1,000 in 2004 to 5.4 $(5.0-5.8)$ in 2005. Fibrate use remained consistently low across the study years, with the lowest rate reported in 2005: $2.2(2.0-2.5)$ per 1,000.

No significant differences by gender were reported among statin users. However, after the health policy intervention, the prevalence of statin use decreased from $40.1(38.6-41.7)$ per 1,000 in 2004 to $37.1(35.6-38.5)$ in 2005 among females, while it rose from $42.0(40.4-43.7)$ to $43.8(42.1-45.5)$ in males in the same period.

With regard to age, prevalence of statin use per year progressively increased with age during the study period, while it tended to decrease in patients over 75 years, and, in particular, over 85 years: $19.7(14.8-26.3)$ per 1,000 in 2005 (data not shown).

Omega-3 fatty acid users were significantly younger (mean age \pm SD: $59.8 \pm 13.3$ years) than statin users (63.8 \pm 11.7 years). After the health policy intervention, however, a strong reduction in omega-3 fatty acid use was reported in all age groups (data not shown).

Among statin users, a subanalysis was performed to assess the annual prevalence of use of individual molecules (Fig. 2). Atorvastatin was the most used statin in each study year [in 2005: $16.3(15.6-17.1)$ per 1,000], followed by simvastatin and pravastatin. One-year prevalence of use of all statins decreased from 2004 to 2005, except for rosuvastatin, which increased from $3.7(3.4-4.1)$ to 6.1 (5.6-6.4).

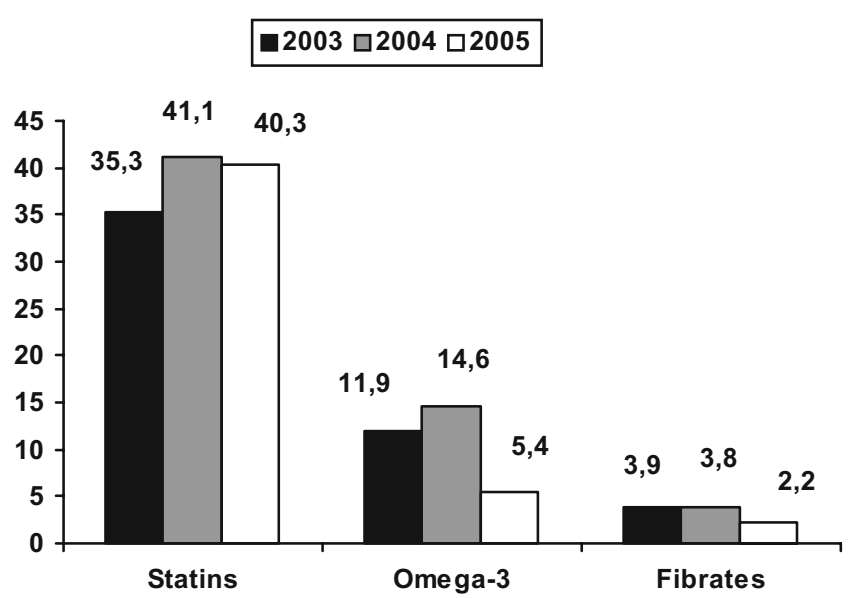

Fig. 1 Prevalence of lipid-lowering drug use per 1,000 inhabitants, stratified by drug type and calendar year 
Fig. 2 Prevalence of statin use per 1,000 inhabitants during the years 2003-2005, stratified by molecule

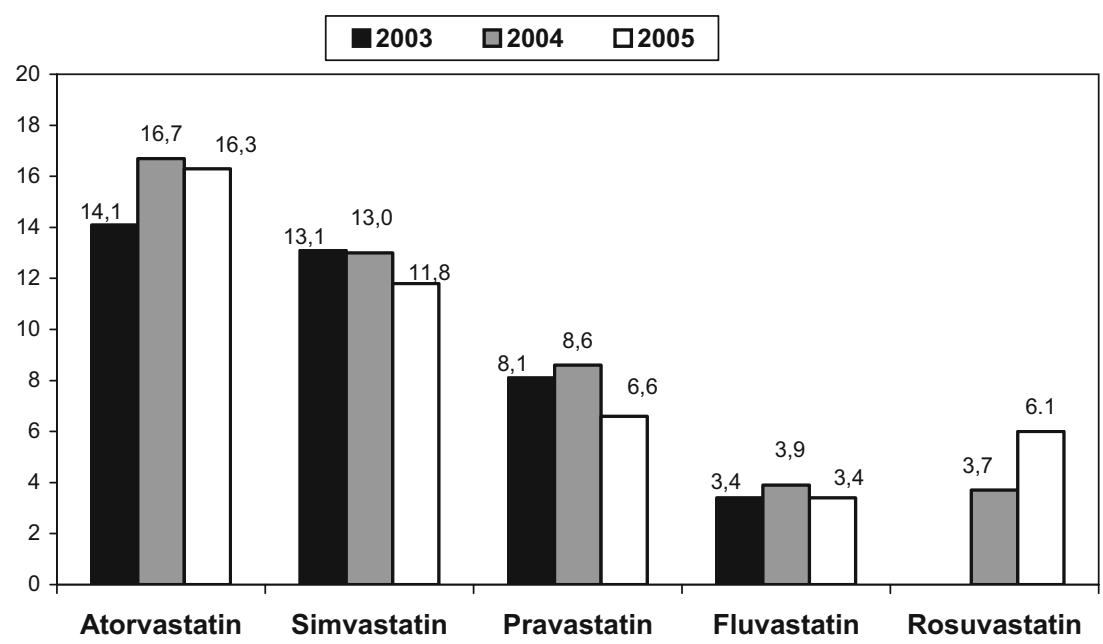

Differences by drug type were highlighted after analyzing the prevalence of use per year, according to indication of use (Fig. 3).

Statin utilization for primary prevention was slightly reduced after reimbursement criteria revision [in 2004: 31.2 (30.2-32.2); in 2005: 27.8 (26.9-28.8)], while a much stronger reduction was reported for omega-3 fatty acid use: from 11.2 (10.6-11.8) in 2004 to 3.8 (3.4-4.1) in 2005.

On the other hand, the rate of statin prescriptions for secondary prevention rose consistently during the study years: from $6.8(6.3-7.2)$ per 1,000 in 2003 up to 12.5 (11.9-13.1) in 2005. In particular, the 1-year prevalence of statin use in patients with diabetes progressively increased from $3.1(2.8-3.5)$ in 2003 to 6.9 (6.4-7.4) in 2005 (data not shown).

In contrast, omega-3 fatty acid use for secondary prevention decreased from $2004(3.4 ; 3.1-3.7)$ to 2005 (1.6; 1.4-1.9). After the health policy intervention, patients

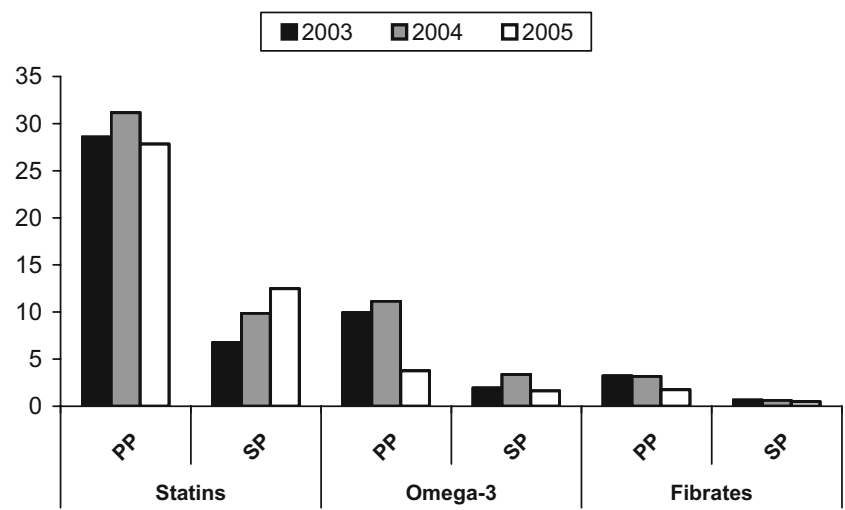

Fig. 3 Prevalence of LLD use per 1,000 inhabitants during the study years, stratified by drug type and indication of use. $P P$ Primary prevention, $S P$ secondary prevention. Secondary prevention was defined as treatment of patients with well-documented coronaropathy, prior stroke, prior myocardial infarction, peripheral obliterative arteriopathy or diabetes affected by myocardial infarction accounted for the majority of users.

Utilization of fibrates remained very low for both primary and secondary prevention compared to other LLDs during the study years.

One-year incidence of LLD use

A significant decrease in annual incidence of LLD treatment was observed from 2004 to 2005 for all drug classes (Fig. 4). However, a highly significant reduction in 1-year incidence was reported only for omega-3 fatty acid users, falling from 5.4 (4.9-5.8) per 1,000 inhabitants in 2004 to $0.9(0.7-1.1)$ in 2005.

Incident treatments with statins (in 2005: 10.1; 9.5-10.7) remained remarkably higher than other LLDs.

\section{Discussion}

In November 2004, the Italian Drug Agency revised LLD reimbursement criteria (Nota 13) in light of updated

$\square 2004 \square 2005$

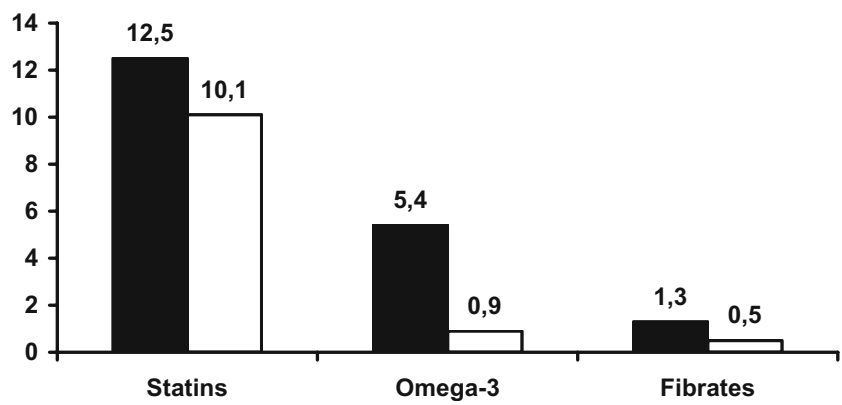

Fig. 4 One-year incidence (per 1,000 inhabitants) of treatment with LLD for the years 2003-2005, stratified by drug type 
scientific evidence [7]. The results of our study highlight that the health policy intervention significantly influenced statin and omega-3 fatty acid use in a general practice in southern Italy.

Concerning statins, a peculiar trend was reported in our analysis: the prevalence of statin use per year increased from 2003 to 2004, while it slightly decreased in 2005 after reimbursement criteria revision. The increase in statin use from 2003 to 2004 is in line with previous investigations that found a constantly growing use of statins in most European countries since their marketing began [14]. In particular, a previous study that explored statin utilization in northern Italy over a 10-year period (1994-2003) reported a $28 \%$ average increase per year [15]. On the other hand, the reimbursement criteria revision led to a slight reduction in both 1-year prevalence and incidence of statin use with a different prescribing pattern of these LLDs.

This could be explained by the fact that statin prescriptions are currently reimbursed for primary prevention according to new cardiovascular risk charts [12]. These risk charts lead to a reduction in statin use as primary prevention since the absolute risk in the Italian population is lower than in the U.S. population. Moreover, this reduction mainly relates to women on the basis of scientific evidence supporting that cardiovascular risk factors affect more men than women $[16,17]$.

The health policy intervention did not determine any significant change in the statin use by age groups, although the time window of the study may have been too short to adequately evaluate it. In all study years, prevalence of use of these LLDs decreased in patients over 75 and, particularly, over 85 years. In line with our results, a British study [18] highlighted that statin use in very old patients is strikingly lower compared to younger patients and, in some cases, the elderly who could benefit from LLD treatment do not receive it. An animated debate is currently underway within the scientific community about the appropriateness of LLD treatment in older patients [19]. Some clinical trials have shown the benefits of statin therapy in cardiovascular prevention also in elderly with hypercholesterolemia [20].

Interestingly, statins were more prescribed for primary than for secondary prevention in all the study years. After reimbursement criteria, however, their use rose in patients for secondary prevention and, in particular, in patients affected by diabetes mellitus. Indeed, patients with hypercholesterolemia can be treated with statins free of charge in case of secondary prevention, including diabetes mellitus, after the health policy intervention.

The revision of reimbursement criteria was based on recent scientific evidence showing a significant reduction in risk of vascular events in diabetic patients who are treated with statins for both primary and secondary prevention $[21,22]$.
Looking at individual molecules, atorvastatin and simvastatin were the most used statins during the study years, in line with a previous European investigation [14] and the Italian national report [6]. In our investigation, however, the annual prevalence of use of any statin was reduced after reimbursement criteria revision, with the exception of rosuvastatin, which was first marketed in Italy in 2004. The extensive marketing campaign launched by the drug manufacturer to promote this statin could partly explain the unusual increase [23]. This finding confirms the trend of newly marketed drugs to be widely prescribed in general practice immediately after their introduction to the drug market, despite a proven lack of effectiveness and safety data derived from clinical practice [24].

The effect of the reimbursement criteria revision on omega-3 fatty acids was a dramatic reduction, in particular of new treatments, from 2004 to 2005. Our results are in line with an Italian national report that reported a $40 \%$ decrease in omega- 3 fatty acid use in 2005 compared to the previous year [6]. This finding might be explained by the restriction in reimbursement criteria of omega-3 fatty acids, which are now free of charge only in patients with a history of myocardial infarction. It should be emphasized that omega-3 prescriptions had been totally reimbursed for primary prevention prior to reimbursement criteria revision, although clinical trials [25] that showed omega-3 fatty acid benefits in CV prevention had included only patients with a recent history of myocardial infarction.

Compared to statins and omega-3 fatty acids, use of fibrates was remarkably low during the observation years. Such a finding is in contrast with other European countries where these LLDs account for almost one-fourth of total LLD utilization [14]. We were not able to accurately characterise the users of fibrates in light of the low utilization of these LLDs.

Overall it seems that the health policy intervention led to changes in LLD prescribing patterns that are in line with Europan recommendations for pharmacological treatment of lipid disorders [26]. On the other hand, a Belgian investigation [27] that analyzed the impact of health policy intervention on statin utilization for secondary prevention highlighted that reimbursement criteria restriction of LLD could also lead to underutilization in patients who would have benefited from LLD therapy.

\section{Strengths and limitations of the study}

To our knowledge, this is the first drug-utilization study targeted to measure the prevalence of LLD use as both primary and secondary prevention in a general practice in southern Italy. In particular, the availability of prescription data from 2003 to 2005 allowed us to evaluate the effect of reimbursement criteria revision on LLD use in clinical 
practice. Moreover, we were also able to evaluate some clinically relevant characteristics of LLD users, such as indication of use.

However, several limitations of our study warrant caution. First of all, we used outpatient prescription data, and we had no information whether LLD prescriptions were actually filled and taken. This limit should be taken into account since around half of the medicines prescribed for people with chronic conditions are not ultimately taken [28]. Nevertheless, our study was aimed at evaluating how the GPs' prescribing behavior was influenced by the health policy intervention. Therefore, this prescription database can be considered suitable for such an investigation. Second, we could not evaluate the number of LLD prescriptions that were not reimbursed by the National Health System. Data from an Italian national report on drug consumption, however, showed a strong decrease in omega-3 fatty acid use after reimbursement criteria revision, thus supporting one of the main findings of our study [29]. Furthermore, we did not take into account familial dyslipidemia as a potential indication of LLD use, even though all LLD treatments are fully reimbursed in such a condition. Indeed, this diagnosis is not accurately codified through ICD-9 codes in general practice, but a previous Italian investigation [30] reported only $13 \%$ of statin prescriptions are filled to patients with familial dyslipidemia.

In conclusion, the health policy intervention modified the trend in the lipid-lowering drug use in general practice. Statin utilization was slightly reduced in 2005 , although it increased in certain categories of patients (diabetes mellitus and secondary prevention). Conversely, omega-3 fatty acid utilization was markedly reduced in 2005 .

In the management of dyslipidemia and cardiovascular prevention, the appropriateness of GP prescribing could be improved through reimbursement criteria revisions based on updated scientific evidence. A definition of these criteria, however, should be developed among regulatory agencies and health professionals.

Acknowledgements We would like to thank all of the following general practitioners from Caserta-1 Local Health Unit who actively participated in this investigation by sending data to the Arianna database in the years 2003-2005: Maria Carmela Abussi, Maria Petronilla Addeo, Ciro Natale Affinita, Luigi Ambrosio, Antonio Anastasio, Maria Clotilde Apperti, Domenico Barbato, Diana Basile, Guido Bernardi, Giuseppe Bernardo, Antonio Betti, Nicola Buono, Anna Campanile, Silvestro Canzano, Maria Concetta Caradonna, Marianna Ceniccola, Augusto Cesare, Maria Teresa Chirico, Angelo Cioffi, Carmine Corbisiero, Alessandro Correra, Pasquale Corvino, Attilio Costarella, Angelo Crescente, Nobile D'acunzo, Rosa D'argenzio, Francesco De Lucia, Franco Pierino De Lucia, Ornella De Matteis, Marcantonio De Rosa, Patrizia De Rosa, Giustino De Sire, Andrea Del Buono, Massimo Del Forno, Renato Del Forno, Giacinto Della Rocca, Annamaria Dell'aquila, Domenico Delle Curti, Angelo Desiato, Marcellino Di Muccio, Giuseppe Diodati, Gianfranco Failli, Umberto Renato Fasulo, Carlo Eugenio Ferrucci, Francesco Ferrucci, Antonio
Gaglione, Giovanni Alfonso Giarrusso, Arturo Gigliofiorito, Agostino Greco, Fernando Iannelli, Enrico Iorio, Maurizio Iuliano, Michele La Vedova, Gennaro Lauritano, Renato Leone, Giuseppe Letizia, Maria Letizia, Innocenzo Lombardi, Antonio Mancino, Antonio Marino, Francesco Carlo Marino, Angelo Marrocco, Giorgio Massara, Bruno Migliozzi, Francesco Mingione, Baldassarre Mirra, Sergio Nunziata, Andrea Pascarella, Domenico Pascarella, Silvio Pascarella, Vincenzo Perone, Manfredo Perrino, M. Giovanna Pontillo, Aldo Porciello, Giovanni Porfidia, Giacomo Lupo Pulcino, Luigi Ragucci, Benedetto Ricciardi, Michele Roberti, Giovanni Russo, Saverio Russo, Clemente Sagnelli, Girolamo Salzillo, Lucia Carla Savignano, Fausto Scalzitti, Antonio Sibillo, Mauro Sicignano, Rodolfo Aniello Sirignano, Giacomo Tartaglione, Massimo Visco, Giacomo Vozza, Francesco Zaccaria, Giovanni Zeppetelli.

Competing interests None of the authors report any conflict of interest.

Funding This study was not supported by any funding.

\section{References}

1. Gotto AM, Grundy SM (1999) Lowering LDL cholesterol: questions from recent meta-analyses and subset analyses of clinical trial data issues from the Interdisciplinary Council on Reducing the Risk for Coronary Heart Disease, 9th Council Meeting. Circulation 99:E1-E7

2. Hebert PR, Gaziano JM, Chan KS, Hennekens CH (1997) Cholesterol lowering with statin drugs, risk of stroke, and total mortality. An overview of randomized trials. JAMA 278:313-321

3. Sever PS, Dahlof B, Poulter NR, Wedel H, Beevers G, Caulfield M, Collins R, Kjeldsen SE, Kristinsson A, McInnes GT, Mehlsen $\mathrm{J}$, Nieminen M, O'Brien E, Ostergren J, ASCOT investigators (2003) Prevention of coronary and stroke events with atorvastatin in hypertensive patients who have average or lower-than-average cholesterol concentrations, in the Anglo-Scandinavian Cardiac Outcomes Trial-Lipid Lowering Arm (ASCOT-LLA): a multicentre randomised controlled trial. Lancet 361:1149-1158

4. Bellosta S, Paoletti R, Corsini A (2004) Safety of statins: focus on clinical pharmacokinetics and drug interactions. Circulation 109: III50-III57

5. Din JN, Newby DE, Flapan AD (2004) Omega 3 fatty acids and cardiovascular disease-fishing for a natural treatment. BMJ 328:30-55

6. Agenzia Italiana del farmaco (AIFA) (2004) L'uso dei farmaci in Italia. Rapporto OsMed 2004. http://www.agenziafarmaco.it/ edi_osmed_2004.html. Accessed 15 Apr 2007

7. Agenzia Italiana del Farmaco (AIFA) (2004) Revisione delle Note CUF. http://www.agenziafarmaco.it/new16_05112004.html. Accessed 15 Apr 2007

8. Lawrenson R, Williams T, Farmer R (1999) Clinical information for research: the use of general practice databases. J Pub Health Med 21:299-304

9. Piacentini N, Trifiró G, Tari M, Moretti S, Arcoraci V, UVEC group (2005) Statin-macrolide interaction risk: a population-based study throughout a general practice database. Eur J Clin Pharmacol 61:615-620

10. Trifiro G, Corrao S, Alacqua M, Moretti S, Tari M, Caputi AP, Arcoraci V, UVEC Group (2006) Interaction risk with proton pump inhibitors in general practice: significant disagreement between different drug-related information sources. Br J Clin Pharmacol 62:582-590 
11. Trifiro G, Barbui C, Spina E, Moretti S, Tari M, Alacqua M, Caputi AP, UVEC group, Arcoraci V (2006) Antidepressant drugs: prevalence, incidence and indication of use in general practice of southern Italy during the years 2003-2004. Pharmacoepidemiol Drug Saf 16:552-559

12. Palmieri L, Panico S, Vanuzzo D, Ferrario M, Pilotto L, Sega R, Cesana G, Giampaoli S, Gruppo di Ricerca del Progetto CUORE (2004) Evaluation of the global cardiovascular absolute risk: the Progetto CUORE individual score. Ann Ist Super Sanita 40:393-399

13. Ferrario M, Chiodini P, Chambless LE, Cesana G, Vanuzzo D, Panico S, Sega R, Pilotto L, Palmieri L, Giampaoli S, CUORE Project Research Group (2005) Prediction of coronary events in a low incidence population. Assessing accuracy of the CUORE Cohort Study prediction equation. Int J Epidemiol 34:413-421

14. Walley T, Folino-Gallo P, Stephens P, Van Ganse E (2005) Trends in prescribing and utilization of statins and other lipid lowering drugs across Europe 1997-2003. Br J Clin Pharmacol 60:543-551

15. Deambrosis P, Saramin C, Terrazzani G, Scaldaferri L, Debetto P, Giusti P, Chinellato A (2007) Evaluation of the prescription and utilization patterns of statins in an Italian local health unit during the period 1994-2003. Eur J Clin Pharmacol 63:197-203

16. Isles CG, Hole DJ, Hawthorne VM, Lever AF (1992) Relation between coronary risk and coronary mortality in women of the Renfrew and Paisley survey: comparison with men. Lancet 339:702-706

17. Savoie I, Kazanjian A (2002) Utilization of lipid-lowering drugs in men and women. A reflection of the research evidence? J Clin Epidemiol 55:95-101

18. DeWilde S, Carey IM, Bremner SA, Richards N, Hilton SR, Cook DG (2003) Evolution of statin prescribing 1994-2001: a case of agism but not of sexism? Heart 89:417-421

19. Ko DT, Mamdani M, Alter DA (2004) Lipid-lowering therapy with statins in high-risk elderly patients: the treatment-risk paradox. JAMA 291:1864-1870

20. LaRosa JC, He J, Vupputuri S (1999) Effect of statins on risk of coronary disease: a meta-analysis of randomized controlled trials. JAMA 282:2340-2346

21. Leiter LA (2005) Diabetic dyslipidaemia: effective management reduces cardiovascular risk. Atheroscler Suppl 6:37-43
22. Vijan S, Hayward RA, American College of Physicians (2004) Pharmacologic lipid-lowering therapy in type 2 diabetes mellitus: background paper for the American College of Physicians. Ann Intern Med 140:650-658

23. Florentinus SR, Heerdink ER, Klungel OH, de Boer A (2004) Should rosuvastatin be withdrawn from the market? Lancet 364:1577

24. Kozyrskyj A, Raymond C, Racher A (2007) Characterizing early prescribers of newly marketed drugs in Canada: a populationbased study. Eur J Clin Pharmacol 63:597-604

25. [No authors listed] (1999) Dietary supplementation with n-3 polyunsaturated fatty acids and vitamin $\mathrm{E}$ after myocardial infarction: results of the GISSI-Prevenzione trial. Gruppo Italiano per lo Studio della Sopravvivenza nell'Infarto miocardico. Lancet 354:447-455

26. De Backer G, Ambrosioni E, Borch-Johnsen K, Brotons C, Cifkova R, Dallongeville J, Ebrahim S, Faergeman O, Graham I, Mancia G, Cats VM, Orth-Gomer K, Perk J, Pyorala K, Rodicio JL, Sans S, Sansoy V, Sechtem U, Silber S, Thomsen T, Wood D, Third Joint Task Force of European and Other Societies on Cardiovascular Disease and Prevention in Clinical Practice (2003) European guidelines on cardiovascular disease prevention in clinical practice. Third Joint Task Force of European and Other Societies on Cardiovascular Disease Prevention in Clinical Practice (constituted by representatives of eight societies and by invited experts). Atherosclerosis 170:145-155

27. Autier P, Creplet J, Vansant G, Brohet C, Paquot N, Muls E, Mullie P, Grivegnee AR (2003) The impact of reimbursement criteria on the appropriateness of 'statin' prescribing. Eur J Cardiovasc Prev Rehabil 10:456-462

28. Jones G (2003) Prescribing and taking medicines. BMJ 327:819

29. Agenzia Italiana del farmaco (AIFA) (2005) L'uso dei farmaci in Italia. Rapporto OsMed 2005. xhttp://www.agenziafarmaco.it/ edi_osmed_2005.html. Accessed 15 Apr 2007

30. Brasesco P, Stimamiglio A, Canepa A, Ghia M, Comaschi M, Bistolfi L, Valle I, Cordano C (2006) La prescrizione di statine in medicina generale. Ricerca nell'ASL-3 Genovese. SIMG 6:18-20. http://www.simg.it/servizi/servizi_riviste2006/06_2006/4.pdf. Accessed 1 May 2007 